\title{
A two-year old boy with ischemic stroke
}

\author{
Mustafa Bolatkale', Çağdaş Can², Ahmet Çağdaş Acara ${ }^{3 *}$ \\ 'Department of Emergency Medicine, Medipol University Hospital, Istanbul, Turkey \\ ${ }^{2}$ Department of Emergency Medicine, Manisa State Hospital, Manisa, Turkey \\ ${ }^{3}$ Department of Emergency Medicine, Bitlis State Hospital, Bitlis, Turkey
}

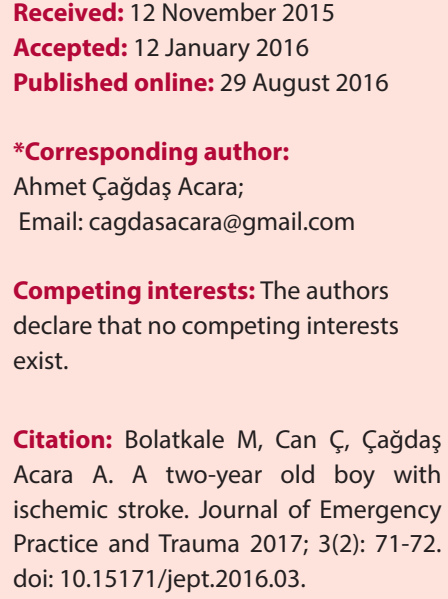

\begin{abstract}
Arterial ischemic stroke (AIS) in adults is considered a serious health threat and requires urgent medical treatment. Prompt diagnosis allows the therapeutic option of thrombolysis within the time window of 3 to 6 hours after first symptoms. Alternatively, early anti-platelet therapy is effective in improving the outcome after stroke. The incidence of pediatric AIS range from 2 to 5 per 100000 children/year. Pediatric AIS has severity and long-term outcomes similar to those in young adults. Two-thirds of children sustaining AIS have neurological deficits that may result in life-long disability, thus critically impacting their potential development. On the other hand 10\%-30\% of the causes of acute strokes are of unknown reasons, therefore careful structural, metabolic and genetic risk factors, requiring more specific treatment, should also be considered in any cases of stroke in children. The diagnosis and treatment should be conducted on the basis of a multidisciplinary approach, including pediatric cardiology, hematology, neurology, neurosurgery and neuroradiology.

Keywords: Ischemic stroke, Children, Prognosis
\end{abstract}

\section{Introduction}

Stroke is the second leading cause of major morbidity and mortality in the world. However, Population-based estimates of the incidence of pediatric arterial ischemic stroke (AIS) range from 2 to 5 per 100000 children per year $(1,2)$. In children, acute ischemic stroke is commonly associated with underlying conditions like congenital or acquired heart disease and sickle cell disease. Cyanotic congenital heart diseases with right-to-left shunt are particularly prone to cause acute ischemic stroke. Though stroke is more common in children with uncorrected congenital heart disease, it occurs even after the correction of the defect $(3,4)$. Besides, $10 \%-30 \%$ of the causes of acute strokes are of unknown reasons.

\section{Case Presentation}

A previously healthy 2 -year-old boy was admitted with acute onset of severe left hemi-paresis. There was no prior history of trauma, infection or family history of migraine or other cerebrovascular diseases. He had fallen on the ground for about 2 hours before admission. He had difficulty in crying. His vital signs were normal and Glasgow coma score (GCS) was 15. Physical examination revealed left facial asymmetry. On motor system evaluation, the patient had power of $2 / 5$ in the left lower limb, $4 / 5$ in the left upper limb and pathologic reflexes were absent. The other organ system physical examinations were within normal limits.
On imaging, non contrast computerized tomography (CT) of the brain was normal. Magnetic resonance imaging (MRI) of the brain revealed acute infarction extending from the left periventricular area to the putamen and splenium. The patient was consulted with the pediatric neurology department (Figure 1A and B). The patient was admitted to the ward with the diagnosis of acute ischemic stroke. His laboratory test values were in normal ranges. Hemoglobin electrophoresis, Factor: II, VII, VIII, XII, fibrinogen and homocysteine levels, antithrombin III and protein CS activities, activated protein $C$ resistance, factor V Leiden mutation panel and MTHFR mutation analysis were normal. Antinuclear antibodies and lupus anticoagulant tests were also negative. The patient was followed up and treated for 20 days. On discharge, he had $5 / 5$ left hemi-paresis and was subsequently enrolled in a physical therapy program.

\section{Discussion}

Even though the general incidence of stroke has decreased, some studies have reported an increase in hospital admissions related to stroke in children and young adults. Although the etiology of acute ischemic stroke in children is different from adults, the current therapeutic approach is based on data obtained from studies conducted on the adult population. This is because there is no sufficient data on the acute treatment and secondary prevention 

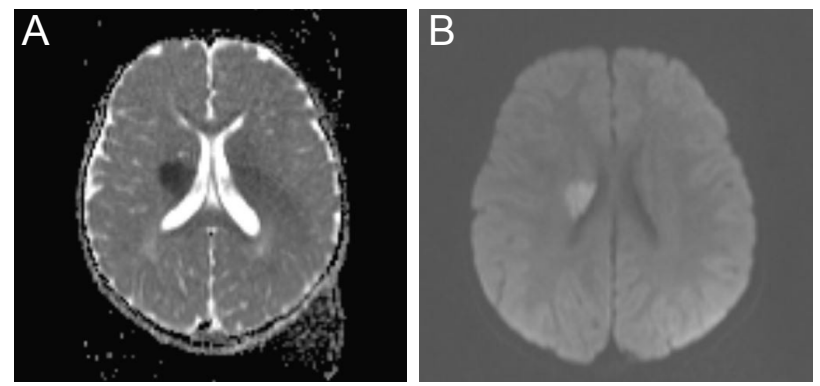

Figure 1. (A) Diffusion MRI shows acute infarction. (B) Shows acute infarction with ADC of diffusion MRI.

after acute ischemic stroke in the pediatric population $(5,6)$. In addition, structural, metabolic and genetic risk factors, requiring more specific treatment, should also be considered in cases of stroke in children. The diagnosis and treatment should be conducted on the basis of a multidisciplinary approach, including pediatric cardiology, hematology, neurology, neurosurgery and neuroradiology (7).

The patient had uncomplicated clinical course. He was discharged on day 21. Prior to his discharge, he regained complete motor strength in all four extremities. Although pediatric stroke is extremely rare, emergency department clinicians should be aware of its existence.

\section{Ethical issues}

This study was carried out in accordance with ethical guidelines and WMA declaration of Helsinki.

\section{Authors' contributions}

All authors contributed to the intellectual planning of the project, intellectual analysis of the data, and writing of the paper.

\section{References}

1. WardlawJM, Murray V, BergeE, DelZoppo GJ.Thrombolysis for acute ischaemic stroke. Cochrane Database Syst Rev 2009;4:CD000213. doi: 10.1002/14651858.CD000213.pub2.

2. Sandercock PA, Counsell C, Gubitz GJ, Tseng MC. Antiplatelet therapy for acute ischaemic stroke. Cochrane Database Sys Rev 2008;3:CD000029. doi: 10.1002/14651858. CD000029.pub2.

3. Steinlin M, Pfister I, Pavlovic J, Everts R, Boltshauser E, Capone Mori A, et al. The first three years of the Swiss Neuropaediatric Stroke Registry (SNPSR): a populationbased study of incidence, symptoms, risk factors and short term outcome. Neuropediatrics 2005; 36(2): 90-7.

4. Bigi S, Fischer U, Wehrli E, Mattle HP, Boltshauser E, Bürki $\mathrm{S}$, et al. Acute ischemic stroke in children versus young adults. Ann Neurol 2011; 70(2): 245-54. doi: 10.1002/ ana.22427.

5. deVeber GA, MacGregor D, Curtis R, Mayank S. Neurologic outcome in survivors of childhood arterial ischemic stroke and sinovenous thrombosis. J Child Neurol 2000; 15(5): 316-24.

6. Amlie-Lefond C, Sébire G, Fullerton HJ. Recent development in childhood arterial ischemic stroke. Lancet Neurol 2008; 7(5): 425-35. doi: 10.1016/S1474-4422(08)70086-3.

7. Marzabadi LR, Shams Vahdati S, Alavi S. Two and a half year old girl with ischemic stroke due to trauma (Lollipop syndrome). Journal of Academic Emergency Medicine 2012; 11(4): 238-40. doi: 10.5152/jaem.2011.068. 